\title{
Sparse Delay-Doppler Image Reconstruction under Off-Grid Problem
}

\author{
Oguzhan Teke \\ Department of Electrical and \\ Electronics Engineering \\ Bilkent University, Turkey \\ Email: teke@ee.bilkent.edu.tr
}

\author{
Ali Cafer Gurbuz \\ Department of Electrical and \\ Electronics Engineering \\ TOBB ETU, Turkey \\ Email: acgurbuz@etu.edu.tr
}

\author{
Orhan Arikan \\ Department of Electrical and \\ Electronics Engineering \\ Bilkent University, Turkey \\ Email: oarikan@ee.bilkent.edu.tr
}

\begin{abstract}
Pulse-Doppler radar has been successfully applied to surveillance and tracking of both moving and stationary targets. For efficient processing of radar returns, delay-Doppler plane is discretized and FFT techniques are employed to compute matched filter output on this discrete grid. However, for targets whose delay-Doppler values do not coincide with the computation grid, the detection performance degrades considerably. Especially for detecting strong and closely spaced targets this causes miss detections and false alarms. Although compressive sensing based techniques provide sparse and high resolution results at subNyquist sampling rates, straightforward application of these techniques is significantly more sensitive to the off-grid problem. Here a novel and OMP based sparse reconstruction technique with parameter perturbation, named as PPOMP, is proposed for robust delay-Doppler radar processing even under the off-grid case. In the proposed technique, the selected dictionary parameters are perturbed towards directions to decrease the orthogonal residual norm. A new performance metric based on KullbackLeibler Divergence (KLD) is proposed to better characterize the error between actual and reconstructed parameter spaces.
\end{abstract}

\section{INTRODUCTION}

In many engineering and science applications the objective is to reconstruct an image or a map of the underlying sensed distribution from available set of measurements. Specifically in radar imaging a spatial map of reflectivity is reconstructed from measurements of scattered electric field. State of the art radar systems operate with large bandwidths or high number of channels which generate very large data sets for processing. On the other hand in most of the radar applications the reflectivity scene consists of small number of strong targets. In both cases, significant amount of data is processed mainly to estimate delay and Doppler of relatively few targets. This point raises the applicability of sparse signal processing techniques for radar signal processing. The emerging field of Compressive Sensing (CS) [1], [2] is a recently developed mathematical framework in which the primary interest is to invert or reconstruct a signal $\boldsymbol{x}$ from noisy linear measurements $\boldsymbol{y}$ in the form $\boldsymbol{y}=\boldsymbol{\Phi} \boldsymbol{x}+\boldsymbol{n}$. The focus of CS is to solve this linear problem in the underdetermined case where number of measurements is less than the number of unknowns which is very important in decreasing the required amount of data to tolerable levels in radar applications.

This work was supported by TUBITAK grant with project number 113E515.

\section{DelaY-DopPler RADAR IMAGing: DATA MODEl AND FORMULATION}

Coherent radar systems transmit a sequence of pulses with known phases and process the received echoes to perform clutter suppression and detection at each angle of interest. Radar transmits $s(t)$, a coherent train of $N_{p}$ pulses:

$$
s(t)=\sum_{i=0}^{N_{p}-1} p\left(t-i T_{P R I}\right) e^{j 2 \pi f_{c} t},
$$

where, $p(t)$ is the individual narrowband pulse waveform, $T_{P R I}$ is the uniform pulse repetition interval and $f_{c}$ is the radar carrier frequency. Assuming $K$ dominant targets with delays of $\tau_{T_{m}}$ and Doppler shifts of $\nu_{T_{m}}, 1 \leq m \leq K$, the received signal following the baseband down-conversion can be expressed as:

$$
y(t)=\sum_{m=1}^{K} \alpha_{m} s\left(t-\tau_{T_{m}}\right) e^{-j 2 \pi \nu_{T_{m}} t}+n(t),
$$

where $\alpha_{m}$ is the complex reflectivity of the individual targets and $n(t)$ is the measurement noise. In compressive sensing formulation, a sampled version of the measurement relation given in (2) is adapted to a linear matrix-vector relationship in delay-Doppler domain. For this purpose 2 dimensional delayDoppler domain which lies in the product space $\left[\tau_{o}, \tau_{f}\right] \times$ $\left[\nu_{o}, \nu_{f}\right]$ must be discretized where $\tau_{0}$ and $\tau_{f}$ are determined by the range and $\nu_{0}$ and $\nu_{f}$ are determined by the velocity of the potential targets. Discretization generates a finite set of $N$ target points $\mathcal{B}=\left\{\boldsymbol{\theta}_{1}, \boldsymbol{\theta}_{2}, \ldots, \boldsymbol{\theta}_{N}\right\}$, where each $\boldsymbol{\theta}_{j}$ representing a grid node of $\left(\tau_{j}, \nu_{j}\right)$. For each grid node $\boldsymbol{\theta}_{j}$ the data model can be calculated as:

$$
\boldsymbol{\psi}_{j}=s\left(\boldsymbol{t}-\tau_{j}\right) \circ \exp ^{-j 2 \pi \nu_{j}} \boldsymbol{t} .
$$

where $\boldsymbol{t} \in \Re^{N_{t}}$ is the vector holding the time samples and operator "o" corresponds to Hadamard product.

Repeating (3) at each $\left(\tau_{j}, \nu_{j}\right)$ generates the dictionary $\mathbf{\Psi}$ where the $j^{t h}$ column of $\boldsymbol{\Psi}$ is $\boldsymbol{\psi}_{j}$. The size of the dictionary $\boldsymbol{\Psi}$ is $N_{t} \times N$ where $N_{t}$ is the number of time samples. If the true target parameters $\tau_{T_{m}}, \nu_{T_{m}}$ falls exactly on the grid points $\left(\tau_{j}, \nu_{j}\right)$ then a linear system of equations can be formed as:

$$
\boldsymbol{y}_{s}=\boldsymbol{\Psi} \boldsymbol{x}+\boldsymbol{n},
$$


where $\boldsymbol{y}_{s}$ is the sampled measurement vector and $\boldsymbol{x}$ is a reflectivity vector defining the delay-Doppler space. If there are $K$ targets in the scene then the vector $\boldsymbol{x}$ should be a $K$ sparse vector.

In the CS formulation, a fraction of the samples obtained at the Nyquist rate carry enough information to represent a sparse signal. Thus a sub-Nyquist sampling can be done and a random subset of $M$ measurements at random times $t_{m}$ can be measured in CS. In general these new measurements can be represented as $\boldsymbol{b}=\boldsymbol{\Phi} \boldsymbol{y}_{s}$ where $\boldsymbol{\Phi}$ is an $M \times N_{t}, M<$ $N_{t}$ measurement matrix constructed by randomly selecting $M$ rows of an $N_{t} \times N_{t}$ identity matrix. The general linear relation is then:

$$
b=\Phi \Psi x+n=A x+n .
$$

The reflectivity vector $\boldsymbol{x}$ is estimated by the solution to the following constrained $\ell_{1}$ minimization problem,

$$
\min _{\boldsymbol{x}}\|\boldsymbol{x}\|_{1} \quad \text { s.t. }\|\boldsymbol{b}-\boldsymbol{A} \boldsymbol{x}\|_{2} \leq \epsilon .
$$

To reduce the computational load, greedy algorithms such as, OMP [3] or CoSamp [4] are also used in many applications. In the following section, the proposed parameter perturbation technique will be introduced within the OMP framework. More details about proposed technique is presented in [5]. For a general non-parametric case, a reconstruction algorithm and some performance bounds are provided in [6].

\section{Parameter Perturbation for Delay-Doppler RECONSTRUCTION}

In general, a target with parameters $\left(\tau_{T}, \nu_{T}\right)$ may not be located at the grid node but is positioned within the grid area with an unknown perturbation from the grid node. Our goal is to perturb the grid parameters and hence the column vectors in $\boldsymbol{A}$, so that a better fit to the measurements can be accomplished.

Here we assume that we are given the grid positions, $\left(\tau_{i}, \nu_{i}\right)$, of the $k$-sparse approximation of the measurement $\boldsymbol{b}$. Running CoSaMP with $k$ or the $k^{t h}$ iteration of the OMP algorithm may serve to this purpose. Perturbation of the given $k$ grid points can be formulated as the following optimization problem:

$$
\begin{array}{r}
\min _{\alpha_{i}, \delta \tau_{i}, \delta \nu_{i}}\left\|\boldsymbol{b}-\sum_{i=1}^{k} \alpha_{i} \boldsymbol{a}\left(\tau_{i}+\delta \tau_{i}, \nu_{i}+\delta \nu_{i}\right)\right\|_{2} \\
\text { s.t. } \quad\left|\delta \tau_{i}\right|<\Delta_{\tau} / 2, \quad\left|\delta \nu_{i}\right|<\Delta_{\nu} / 2,
\end{array}
$$

where $\alpha_{i}$ corresponds to representation coefficient, $\boldsymbol{a}\left(\boldsymbol{\theta}_{i}\right)$ is the data model with parameters $\boldsymbol{\theta}_{i}$ and $\left(\delta \tau_{i}, \delta \nu_{i}\right)$ corresponds to perturbation of the $i^{t h}$ target. Assume that there exist a solver for the problem in (7), namely $\mathbb{S}(\cdot)$. This solver takes the measurements $\boldsymbol{b}$ and the $k$ grid points and returns the representation coefficients and perturbations. In an abstract sense, this solver can be written as:

$$
\left(\boldsymbol{\alpha},\left[\delta \boldsymbol{\theta}_{1}, \ldots, \delta \boldsymbol{\theta}_{N}\right]\right)=\mathbb{S}\left(\boldsymbol{b},\left[\boldsymbol{\theta}_{1}, \ldots, \boldsymbol{\theta}_{N}\right]\right) .
$$

When such a solver in (8) is utilized in OMP iterations, an "ideal" parameter perturbed OMP (I-PPOMP) algorithm, which is provided in Table I, can be implemented. Note that this solver is independent from OMP and can be utilized within any algorithm that provides a $k$-sparse representation. We prefer OMP due to its simplicity.

$$
\text { TABLE I }
$$

Ideal Parameter Perturbed-OMP (I-PPOMP) Algorithm

\begin{tabular}{l}
\hline \hline Inputs: $(\boldsymbol{A}, \boldsymbol{b}, \epsilon)$ \\
\hline Initialization: \\
$\boldsymbol{b}_{\perp, 0}=\boldsymbol{b}, \boldsymbol{T}_{0}=\{\}, e=\left\|\boldsymbol{b}_{\perp, 0}\right\|_{2}, k=1$ \\
\hline Keep iterating until $e<\epsilon$ \\
$j^{*}=\arg \max _{1 \leq j \leq N}\left|\boldsymbol{a}\left(\boldsymbol{\theta}_{j}\right)^{H} \boldsymbol{b}_{\perp, k-1}\right|$ \\
$\boldsymbol{T}_{k}=\boldsymbol{T}_{k-1} \bigcup\left\{\boldsymbol{\theta}_{j^{*}}\right\}$ \\
$\left(\boldsymbol{\alpha},\left[\delta \boldsymbol{\theta}_{1} \ldots \delta \boldsymbol{\theta}_{k}\right]\right)=\mathbb{S}\left(\boldsymbol{b}, \boldsymbol{T}_{k}\right)$ \\
$\boldsymbol{b}_{\perp, k}=\boldsymbol{b}-\sum_{i=1}^{k} \alpha_{i} \boldsymbol{a}\left(\boldsymbol{\theta}_{i}+\delta \boldsymbol{\theta}_{i}\right)$ \\
$e=\left\|\boldsymbol{b}_{\perp, k}\right\|_{2}$ \\
$k=k+1$ \\
\hline Output: $\left(\boldsymbol{\alpha},\left[\delta \boldsymbol{\theta}_{1} \ldots \delta \boldsymbol{\theta}_{k}\right], \boldsymbol{T}_{k}\right)$ \\
\hline \hline
\end{tabular}

Since the required optimization is non-convex, here we propose to use a gradient descent optimization of the cost function. Therefore starting from the grid nodes, the columns of $\boldsymbol{A}$ will be gradually perturbed until a convergence criteria is met. To simplify the iterations further $\alpha_{i}$ 's and $\delta \boldsymbol{\theta}_{i}=\left(\delta \tau_{i}, \delta \nu_{i}\right)$ 's will be sequentially updated in the following way:

First initialize $\boldsymbol{\theta}_{i, 1}=\boldsymbol{\theta}_{i}=\left(\tau_{i}, \nu_{i}\right), i=1, \ldots, k$, to grid centers and obtain the representation coefficients $\boldsymbol{\alpha}_{1}$ as:

$$
\boldsymbol{\alpha}_{1}=\arg \min _{\boldsymbol{\alpha}}\left\|\boldsymbol{b}-\sum_{i=1}^{k} \alpha_{i} \boldsymbol{a}\left(\boldsymbol{\theta}_{i, 1}\right)\right\|_{2} .
$$

Starting from $l=1$, until convergence, perform updates:

$$
\boldsymbol{\theta}_{i, l+1}=\boldsymbol{\theta}_{i, l}+\delta \boldsymbol{\theta}_{i, l},
$$

where $l$ represents the perturbation iteration, $i$ represents the target index and $\delta \boldsymbol{\theta}_{i, l}$ are found as the solution of the following

$$
\begin{aligned}
& \arg \min _{\substack{\delta \tau_{i}:\left|\delta \tau_{i}\right| \leq \Delta_{\tau} / 2 \\
\delta \nu_{i}:\left|\delta \nu_{i}\right| \leq \Delta_{\nu} / 2}}\left\|\boldsymbol{b}-\sum_{i=1}^{k} \alpha_{i, l} \boldsymbol{a}\left(\tau_{i, l}+\delta \tau_{i}, \nu_{i, l}+\delta \nu_{i}\right)\right\|_{2} \text {, } \\
& \boldsymbol{\alpha}_{l}=\arg \min _{\boldsymbol{\alpha}}\left\|\boldsymbol{b}-\sum_{i=1}^{k} \alpha_{i} \boldsymbol{a}\left(\boldsymbol{\theta}_{i, l}\right)\right\|_{2}
\end{aligned}
$$

The problem defined in (11) is a standard least squares (LS) formulation, however obtaining solution to the constrained nonlinear optimization problem in (10) is not practical for radar applications. Linearization of the cost function in (10) around $\boldsymbol{\theta}_{i, l}=\left(\tau_{i, l}, \nu_{i, l}\right)$ significantly reduces the complexity of the optimization. For this purpose, $\boldsymbol{a}\left(\tau_{i, l}+\delta \tau_{i}, \nu_{i, l}+\delta \nu_{i}\right)$ can be approximated by using the first order Taylor series as: $\boldsymbol{a}\left(\tau_{i, l}+\delta \tau_{i}, \nu_{i, l}+\delta \nu_{i}\right) \approx \boldsymbol{a}\left(\tau_{i, l}, \nu_{i, l}\right)+\frac{\partial \boldsymbol{a}}{\partial \tau_{i, l}} \delta \tau_{i}+\frac{\partial \boldsymbol{a}}{\partial \nu_{i, l}} \delta \nu_{i}$.

By using this expansion, and ignoring the constraints on the perturbations, problem in (10) can be re-written as:

$$
\left[\delta \boldsymbol{\theta}_{1, l} \ldots \delta \boldsymbol{\theta}_{k, l}\right]=\arg \min _{\boldsymbol{u}}\left\|\boldsymbol{r}_{l}-\boldsymbol{B}_{l} \boldsymbol{u}\right\|_{2}
$$

where $\boldsymbol{r}_{l}=\boldsymbol{b}-\sum_{i=1}^{k} \alpha_{i, l} \boldsymbol{a}\left(\boldsymbol{\theta}_{i, l}\right)$ is the orthogonal residual from the least squares in (11), $\boldsymbol{B}_{l} \in \mathcal{C}^{M \times 2 k}$ is the matrix 
holding the weighted partial derivatives at the linearization point and is defined as:

$$
\boldsymbol{B}_{l}=\left[\ldots, \Delta_{\tau} \alpha_{i, l} \frac{\partial \boldsymbol{a}}{\partial \tau_{i, l}}, \ldots, \ldots, \Delta_{\nu} \alpha_{i, l} \frac{\partial \boldsymbol{a}}{\partial \nu_{i, l}}, \ldots\right],
$$

and $\boldsymbol{u}=\left[\delta \tau_{1}, \ldots \delta \tau_{k}, \delta \nu_{1}, \ldots \delta \nu_{k}\right]^{T} \in \mathcal{R}^{2 k \times 1}$ is the dummy vector variable containing updates in the $l^{\text {th }}$ iteration on the corresponding parameters. Each partial derivative in $\boldsymbol{B}_{l}$ is scaled by its corresponding grid size so that corresponding updates become unitless. Notice that $\boldsymbol{B}_{l}$ is different in each iteration and a new linearization is made at each updated parameter point.

We adapt a gradient descent type algorithm to solve (12) and take a small step in the direction of negative gradient. Then the new parameter point will be used in the next iteration and so on until the convergence. Let $J(\boldsymbol{u})=\left\|\boldsymbol{r}_{l}-\boldsymbol{B}_{l} \boldsymbol{u}\right\|_{2}^{2}$ and negative of the gradient of $J$ at the linearization point will be $\left.J(\boldsymbol{u})\right|_{\boldsymbol{u}=\mathbf{0}}=2 \boldsymbol{B}_{l}^{H} \boldsymbol{r}_{l}$. When solution is forced to be real, step direction is found to be as $\operatorname{Re}\{-\nabla \boldsymbol{u} J(\boldsymbol{u}) \mid \boldsymbol{u}=0\}=$ $\operatorname{Re}\left\{2 \boldsymbol{B}_{l}^{H} \boldsymbol{r}_{l}\right\}$. As a result, alternating gradient descend solution of the main problem in (7) can be written as;

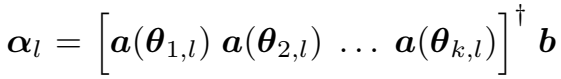

$$
\begin{aligned}
& \boldsymbol{\theta}_{i, l+1}=\boldsymbol{\theta}_{i, l}+\boldsymbol{\mu}_{i, l} \operatorname{Re}\left\{\boldsymbol{B}_{l}^{H} \boldsymbol{r}_{l}\right\}
\end{aligned}
$$

where $\boldsymbol{\mu}_{i, l}$ is the step size. To satisfy the constraints in (10) and keep the updated points within the grid, the algorithm will also check that the total perturbations will not exceed the grid size at each iteration. Equation (13) defines the main update iterations of the proposed gradient based perturbation solver (GS) - $\widehat{\mathbb{S}}(\cdot)$ for (7) which is summarized in Table II. Notice that, when $\mathbb{S}(\cdot)$ in Table I is replaced with the $\widehat{\mathbb{S}}(\cdot)$, proposed PPOMP algorithm is obtained.

TABLE II

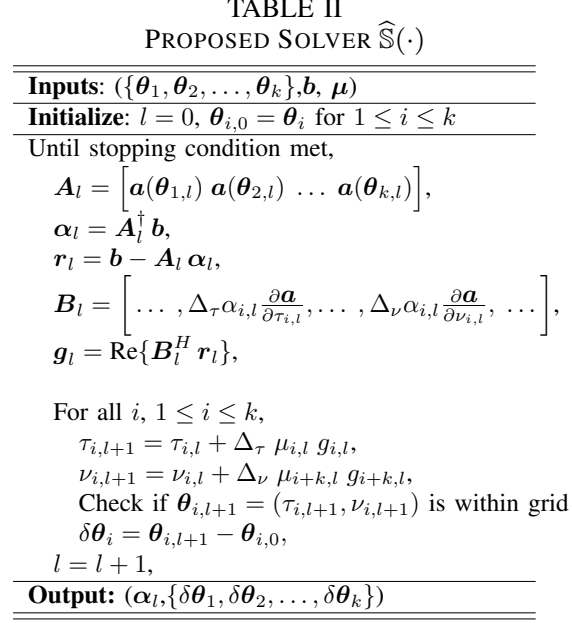

For the selection of step size $\mu$ there are several possibilities. If the gradient of a function is Lipschitz continuous with a constant $L$, gradient descent steps converges to a local optima by using constant step size that satisfies $\mu<2 / L$ [7], [8]. As shown in [5], normalized form of the non-linear objective function in (10) is Lipschitz continuous with $L=10 \pi^{2}$. In the presented results, step size is selected selected as $\mu_{i, l} \leq$
$0.01<2 / L$ and decreases throughout the iterations, thus our selection of the step size is guaranteed to converge to a local minima.

For the pulse-Doppler radar application, the gradient computations simplify further requiring only component-wise multiplication of vectors that has $M$ multiplications each. Hence $\boldsymbol{B}_{l}$ can be computed efficiently and the total computational complexity of PPOMP will be in the same order as OMP algorithm due to mainly solution of least squares in both techniques.

\section{Simulation Results}

In the simulations, a classical single receiver-single transmitter pulsed-Doppler radar transmitting a linear chirp signal $p(t)$ with bandwidth of $B=1.5 \mathrm{MHz}$ and pulse width of $T_{p}=20 \mu \mathrm{s}$ is considered. In the coherent processing, a pulse train of $N_{p}=8$ pulses are used with $T_{P R I}=$ $50 \mu s$. The delay and Doppler space is chosen as the maximum unambiguous ranges of $\left[T_{p}, T_{P R I}-T_{p}\right]$ in delay and $\left[-1 /\left(2 T_{P R I}\right), 1 /\left(2 T_{P R I}\right)\right]$ in Doppler. To create the forward linear model the space is discretized to grids with Rayleigh resolution spacing in both parameter axis which is $\Delta_{\nu}=1 /\left(N_{p} T_{P R I}\right)$ in Doppler and $\Delta_{\tau}=1 /(2 B)$ in delay. For the simulated case this discretization creates a total of $N=279$ grid nodes. Sparse target scene is modeled as $K=9$ point reflectors that are generated with delay and Doppler parameters randomly selected from the defined continuous delay-Doppler space where none of them exactly coincides with the chosen grid nodes. The complex reflectivity of the parameters are selected randomly with magnitudes selected from a normal distribution of $N(5,1)$ and phases selected uniformly from $[0,2 \pi]$. For $M=2 N / 3=186$ randomly spaced time samples in $\left[0, N_{p} T_{P R I}\right]$, the received signal is computed using (2). If the samples are taken at the Nyquist rate, total number of samples is $\left(N_{p} T_{P R I}\right)(2 B)=1200$. Therefore $M$ corresponds to only $15 \%$ of the Nyquist rate samples. Measurement noise corresponding to an SNR of 25 $\mathrm{dB}$ is added to the computed time samples.

The actual target reflectivity and its reconstruction by the proposed PPOMP technique are shown in Figure 1(a) and (b), respectively. Even the targets are off the grid, PPOMP could provide accurate reconstruction of the sparse target scene. Note that PPOMP doesn't have any prior information about the actual sparsity level. OMP technique using the same measurements and the same termination criteria with PPOMP generated the result shown in Fig. 1(c). Due to off grid targets OMP generates large number of significant peaks resulting in excessively many false target detections even at high level of detection threshold.

Fig. 2(a) shows the gradient based steps taken for one of the targets starting from the grid center. With decreasing step sizes, the algorithm converges to the actual target parameters. Similarly 2(b) shows gradient steps taken for two closely spaced targets. Separation of these two targets is closer than the grid size corresponding to the classical Rayleigh resolution limit both in delay and Doppler axis. While a matched filter won't 


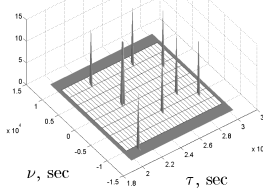

(a)

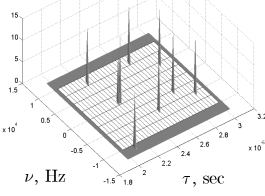

(b)

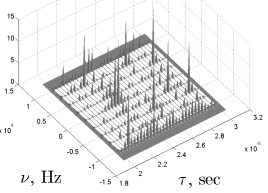

(c)
Fig. 1. (a) True delay-Doppler space reflectivity with $K=9$ off the grid targets, (b) PPOMP reconstruction result, (OMP) reconstruction result

be able to resolve these two targets, the proposed PPOMP technique could identify their actual positions accurately. This shows the high resolution capability of the proposed PPOMP technique.

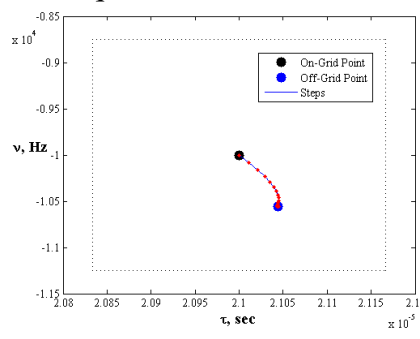

(a)

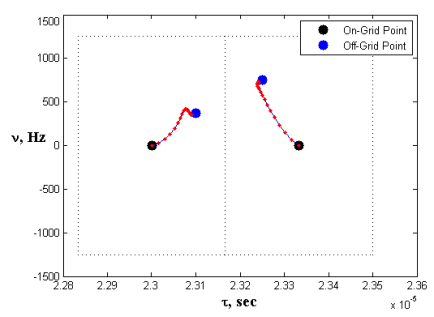

(b)
Fig. 2. Gradient based steps taken within the PPOMP algorithm at (a) one of the target grids, with (b) two targets grids where the two target parameters are closer than a grid size in both $\tau$ and $\nu$.

One of the important problems of standard CS based reconstruction techniques is that in the presence of off-grid targets, they tend to generate a non-sparse reconstruction. When sparsity levels of the actual and reconstructed signals do not match, classical error criterions become inappropriate. Here, we propose to use Kullback-Leibler Divergence(KLD) between the actual and reconstructed target scenes, which is detailed in [5].

To illustrate the performance of the proposed technique, PPOMP is compared with the oracle solver, standard OMP, $\ell_{1}$ reconstruction and AA-P-BPDN algorithm proposed in [9] with different sparsity levels. The average KLD for each technique is provided in Fig. 3. Oracle solution is the least squares on the actual target points and grid-oracle is the LS on the corresponding grid points.

In Fig. 3(a), where the corresponding grid points are known apriori, gradient solver performs very close to the oracle solution compared to AA-P-BPDN. In Fig. 3(b) performance of the results obtained in the absence of the knowledge of corresponding grid points, is shown. PPOMP is inferior to only the gradient solver which requires the knowledge of the corresponding grid points. For comparison purposes, results of OMP operating in a finer grid is also shown in Fig. 3(b). As it was reported in [6], use of finer grids in OMP does not provide comparable performance to the proposed PPOMP.

\section{CONCLUSiON}

In this paper a novel compressive sensing technique is proposed to alleviate the issues related with the reconstruction of the targets whose positions do not coincide with the assumed delay-Doppler grid. The proposed PPOMP technique adapts the signal dictionary to the actual measurements by

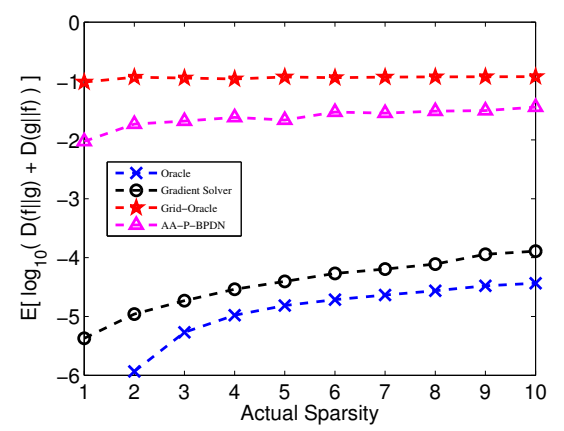

(a)

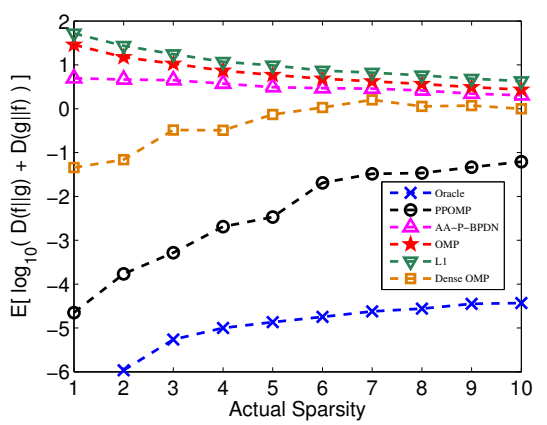

(b)

Fig. 3. Kullback-Liebler Divergences between the correct and reconstructed target scenes as a function of actual sparsity level. (a) Performance of the gradient solver. (b) Performance of the proposed PPOMP algorithm.

performing perturbations of the parameters governing the signal dictionary. To quantify the performance, KullbackLiebler Divergence is proposed as the error metric for off-grid target reconstruction performance comparisons. Compared to the standard OMP technique, proposed method provides significantly lower errors for a wide range of sparsity levels. Furthermore, due to the lower complexity of its implementation, PPOMP technique is more feasible in radar applications than the convex optimization based techniques.

\section{REFERENCES}

[1] D. Donoho, "Compressed sensing," IEEE Trans. Information Theory, vol. 52, no. 4, pp. 1289-1306, 2006.

[2] E. Candes, J. Romberg, and T. Tao, "Robust uncertanity principles: Exact signal reconstruction from highly incomplete frequency information," IEEE Trans. Information Theory, vol. 52, pp. 489-509, 2006.

[3] J. Tropp and A. Gilbert, "Signal recovery from random measurements via orthogonal matching pursuit," IEEE Trans. Information Theory, vol. 53, no. 12, pp. 4655-4666, Dec. 2007.

[4] D. Needell and J. A. Tropp, "CoSaMP: Iterative signal recovery from incomplete and inaccurate samples," Appl. Comp. Harmonic Anal., vol. 26, no. 3, pp. 301-321, 2009.

[5] O. Teke, A. C. Gurbuz, and O. Arikan, "A robust compressive sensing based technique for reconstruction of sparse radar scenes," Digital Signal Processing, vol. 27, pp. 23-32, 2014.

[6] O. Teke, A. Gurbuz, and O. Arikan, "Perturbed orthogonal matching pursuit," Signal Processing, IEEE Transactions on, vol. 61, no. 24, pp. 6220-6231, 2013.

[7] S. Boyd and L. Vandenberghe, Convex Optimization. Cambridge University Press, 2004.

[8] D. P. Bertsekas, Nonlinear Programming. Athena Scientific, 1999.

[9] Z. Yang, C. Zhang, and L. Xie, "Robustly stable signal recovery in compressed sensing with structured matrix perturbation," Signal Processing, IEEE Transactions on, vol. 60, no. 9, pp. 4658-4671, 2012. 